\title{
Is new-onset postoperative atrial fibrillation a benign complication?
}

\author{
Manuel J. Antunes, MD, PhD, DSc
}

\author{
From the Center of Cardiothoracic Surgery, University Hospital and Faculty of Medicine, Coimbra, Portugal. \\ Disclosures: Author has nothing to disclose with regard to commercial support. \\ Received for publication March 13, 2017; accepted for publication March 17, 2017; available ahead of print April \\ $12,2017$. \\ Address for reprints: Manuel J. Antunes, MD, PhD, DSc, Center of Cardiothoracic Surgery, Centro Hospitalar \\ Universitário de Coimbra, Praceta Professor Mota-Pinto, 3000-075 Coimbra, Portugal (E-mail: antunes.cct. \\ chuc@sapo.pt). \\ J Thorac Cardiovasc Surg 2017;154:490-1 \\ $0022-5223 / \$ 36.00$ \\ Copyright (c) 2017 by The American Association for Thoracic Surgery \\ http://dx.doi.org/10.1016/j.jtcvs.2017.03.068
}

New-onset atrial fibrillation (AF), that is, AF occurring in patients without a history of AF, is most common in the hospital setting after cardiac surgery in general and valve surgery in particular. The incidence after valve surgery has been described to be as high as $50 \%$. AF occurs more frequently in older patients and in those with mitral valve disease, but it also occurs in young patients and after aortic valve surgery and coronary revascularization. ${ }^{1,2}$ Previous history of AF, older age, hypertension, left ventricular hypertrophy, and congestive heart failure, as well as prolonged aortic crossclamp and pump perfusion times, have often been associated to the occurrence of postoperative (within the same hospital admission) AF after cardiac surgery. As judged from the literature, the type of surgery (aortic vs mitral vs coronary) is probably also a risk factor. ${ }^{3,4}$ In this respect, it is important to stress that the incidence of new-onset AF is much lower after transcatheter aortic valve implantation than after surgical aortic valve replacement (AVR), ${ }^{2,4,5}$ which may implicate cardiopulmonary bypass and cardioplegia as triggering factors.

This complication is said to effect early and late patient survival, independently of the treatment, 6,7 and several protocols for prophylaxis against this arrhythmia have been proposed but are not consistently applied by a large number, perhaps the majority, of surgical units. The adverse effect on long-term survival may not be related to the short-lived new-onset AF but rather to the underlying pathology associated to the arrhythmia, especially pathology that affects the myocardium, principally in atherosclerotic coronary artery disease.

It is not survival alone, however, that should be cause for concern; AF, even in episodes of limited duration, may result in transient ischemic attacks, ischemic or hemorrhagic strokes, and peripheral thromboembolism, which is why affected patients should immediately be anticoagulated. ${ }^{8,9}$

In an article in this issue of the Journal, Swinkels and colleagues $^{10}$ from The Netherlands analyze the effect on long-term survival (as late as 20 years; mean of 18 years)

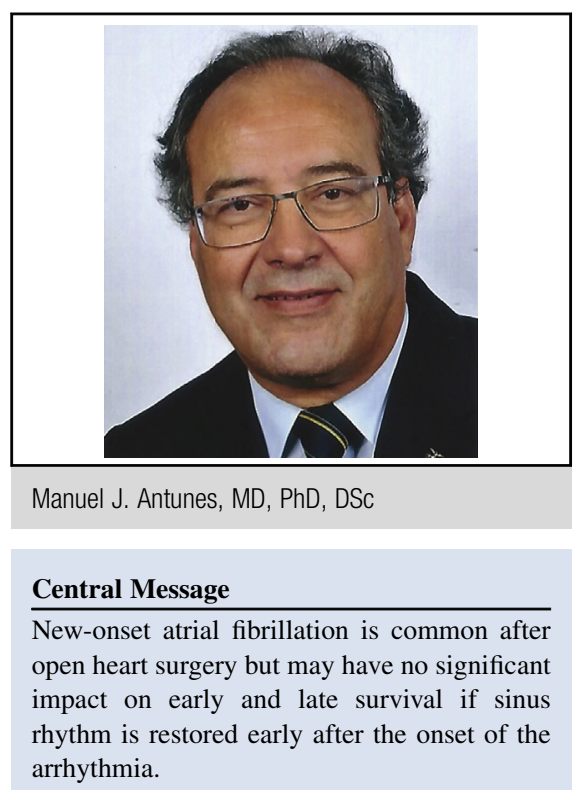

See Article page 492.

of new-onset postoperative AF after aortic valve replacement, which occurred in $42.4 \%$ of their patients. Swinkels and colleagues ${ }^{10}$ conclude that "new-onset postoperative AF after AVR does not affect long-term survival when treatment is aimed to restore sinus rhythm before discharge home." Their consequent message is that patients who have new-onset AF after cardiac surgery should not leave the hospital before correction of the arrhythmia and restoration of sinus rhythm, within 24 to 48 hours from onset, by medication and when medication fails by direct-current cardioversion, which is now (but was not then) recommended by the guidelines.

I believe this to be, by far, the longest follow-up of a series of patients with new-onset postoperative AF after cardiac surgery, and also a series in which a definitive and rigid protocol of early treatment of the AF was in practice, which may have made all the difference. This study is at odds with previously published studies, however, with opposite conclusions. Filardo and coworkers ${ }^{6}$ reported that new-onset post-AVR AF is significantly associated with increased long-term risk of mortality independent of the preoperative severity of disease. They found that the risk of long-term mortality in patients who had new-onset post-AVR AF was $48 \%$ higher than that in patients without it. Levy and colleagues" $^{7}$ also found that "post-operative AF incidence 
in aortic valve replacement is high and correlates with age in patients aged 70 years and older and significantly more pronounced in patients aged 80 years." In their experience, the intensive care unit and hospital stays were longer, but there was no increase in mortality or stroke.

Swinkels and colleagues ${ }^{10}$ believe that one of the reasons for the discrepancy was the homogeneous character of their series, composed as it was almost entirely of patients who had isolated AVR, especially because the previously mentioned risk factors were not more prevalent in their new-onset AF group. They also consider their long follow-up, almost double those reported by others, to be a possible factor in the results, although it is highly unlikely that the effect of 1 or a few short-lived early episodes could have an impact on survival beyond the first decade after surgery.

Another important aspect to consider is that the antiarrhythmic drugs used prophylactically or therapeutically at the time of treatment of this patient cohort (1990-94) are no longer used or have been replaced by new and more efficacious pharmacologic agents. ${ }^{9}$

In conclusion, this contribution from Swinkels and colleagues ${ }^{10}$ reassures us that new-onset $\mathrm{AF}$, common after heart surgery, may have no significant impact on early and late survival if sinus rhythm is effectively and permanently restored early after the onset of the arrhythmia and before the patient's discharge from the hospital.

\section{References}

1. Pivatto Júnior F, Teixeira Filho GF, Sant'anna JR, Py PM, Prates PR, Nesralla IA, et al. Advanced age and incidence of atrial fibrillation in the postoperative period of aortic valve replacement. Rev Bras Cir Cardiovasc. 2014;29:45-50.

2. Tanawuttiwat T, O'Neill BP, Cohen MG, Chinthakanan O, Heldman AW, Martinez CA, et al. New-onset atrial fibrillation after aortic valve replacement: Comparison of transfemoral, transapical, transaortic, and surgical approaches. J Am Coll Cardiol. 2014;63:1510-9.

3. Girerd N, Magne J, Pibarot P, Voisine P, Dagenais F, Mathieu P. Postoperative atrial fibrillation predicts long-term survival after aortic-valve surgery but not after mitral-valve surgery: a retrospective study. BMJ Open. 2011;1:e000385.

4. Jørgensen TH, Thygesen JB, Thyregod HG, Svendsen JH, Søndergaard L. New-onset atrial fibrillation after surgical aortic valve replacement and transcatheter aortic valve implantation: a concise review. J Invasive Cardiol. 2015; 27:41-7.

5. Tarantini G, Mojoli M, Windecker S, Wendler O, Lefèvre T, Saia F, et al. Prevalence and impact of atrial fibrillation in patients with severe aortic stenosis undergoing transcatheter aortic valve replacement: an analysis from the SOURCE XT Prospective Multicenter Registry. JACC Cardiovasc Interv. 2016;9:937-46.

6. Filardo G, Hamilton C, Hamman B, Hebeler RF Jr, Adams J, Grayburn P. Newonset postoperative atrial fibrillation and long-term survival after aortic valve replacement surgery. Ann Thorac Surg. 2010;90:474-9.

7. Levy F, Rusinaru D, Maréchaux S, Charles V, Peltier M, Tribouilloy C. Determinants and prognosis of atrial fibrillation in patients with aortic stenosis. Am J Cardiol. 2015;116:1541-6.

8. Camm AJ, Lip GY, De Caterina R, Savelieva I, Atar D, Hohnloser SH, et al; Document Reviewers. 2012 Focused update of the ESC guidelines for the management of atrial fibrillation: An update of the 2010 ESC guidelines for the management of atrial fibrillation-developed with the special contribution of the European Heart Rhythm Association. Europace. 2012;14:1385-413.

9. Di Biase L. Use of direct oral anticoagulants in patients with atrial fibrillation and valvular heart lesions. J Am Heart Assoc. 2016;5:e002776.

10. Swinkels BM, de Mol BA, Kelder JC, Vermeulen FE, ten Berg JM. New-onset postoperative atrial fibrillation after aortic valve replacement: effect on longterm survival. J Thorac Cardiovasc Surg. 2017;154:492-8. 